\title{
A Multi-loading, Climate-Controlled, Stationary ROI Device for In-Situ X-ray CT Hygro-Thermo-Mechanical Testing
}

\author{
N.H. Vonk ${ }^{1}$ - E.C.A. Dekkers ${ }^{2}$ - M.P.F.H.L. van Maris ${ }^{1}$ - J.P.M. Hoefnagels ${ }^{1}$ iD
}

Received: 31 May 2018 / Accepted: 24 August 2018 / Published online: 12 October 2018

(C) The Author(s) 2018

\begin{abstract}
In-situ CT mechanical testing yields a full 3D description of a sample material's behaviour under specific loads. In the literature various devices are proposed which enable in-situ CT hygro-, thermo- or mechanical testing, each with its own merits and limitations. However none of them is able to perform advanced hygro-thermo-mechanical tests on specimens subjected to multiple loading modes, while accurately controlling and measuring the force, displacement, temperature and relative humidity in real time. Therefore, this work proposes an in-situ CT device which allows such multi-faceted experiments. Improvements to the current state-of-the-art devices include: (1) a compact, lightweight and rotationally symmetric design that enables high-resolution CT scans by minimization of wobble during scanning, in practically all labscale CT scanners; (2) a stationary region of interest by loading the sample from both sides, which enables high resolution CT characterization of materials exhibiting a large fracture strain; and (3) improved testing modularity by exchanging clamping methods to allow samples of various sizes (e.g., circular or rectangular) to be inserted in a variety of ways, thereby facilitating complex experiments such as three- or four-point bending tests. Validation experiments demonstrate that stringent requirements on CT resolution, loading and displacement accuracy and climate control are met. Furthermore, the in-situ testing capabilities of the device were validated by CT characterization of the creasing and folding process of multi-layer cardboard under varying (controlled) levels of relative humidity and temperature.
\end{abstract}

Keywords In-situ mechanical testing · X-ray computed tomography $\cdot$ Climate control $\cdot$ Multi Loading $\cdot$ Stationary ROI . Micro-mechanics

\section{Introduction}

$\mathrm{X}$-ray Computed Tomography (CT) is a powerful nondestructive technique used to obtain high-resolution 3D description of samples. The technique is widely used on samples which have critical intrinsic features such as composite materials, biological (bone) samples, fibre networks, complex 3D-printed structures, etc. In the past decade, however, a growing interest has arisen in performing mechanical tests in combination with CT scanning. Initially, experiments were performed by deforming the specimen ex-situ and subsequently scanning

J.P.M. Hoefnagels

J.P.M.Hoefnagels@tue.nl

1 Department of Mechanical Engineering, Eindhoven University of Technology, Eindhoven, The Netherlands

2 Equipment and Prototyping Centre, Eindhoven University of Technology, Eindhoven, The Netherlands it in the CT scanner [1, 2]. Obviously, convenience lies with an in-situ approach, where the intrinsic 3D deformation can directly be linked to the mechanical response. To this end, a number of in-situ (thermo)mechanical test devices have been developed [3-10], each with different limits and choices in terms of loading modes (tension, compression, bending, creep, etc.), load and deformation range and resolution, CT resolution and, for some devices, temperature and/or relative humidity range. While each of these devices has its own merits, with none of them it is possible to perform hygro-thermomechanical testing of materials under multiple loading modes with accurate, real-time measurement of the force and displacement in a controlled environment. Combining all these characteristics in a single in-situ CT testing device would enable advanced hygro-thermo-mechanical experimentation, such as time-dependent hygro-expansion characterization of paper under tension [11], three- and four-point bending tests of cardboard [12] under different temperatures, delamination of Carbon-Fibre Reinforced 
Polymers (CFRP) under compression [13] and a stiffness analysis of bone tissue under compression [14], whereas these experiments [11-14] were so far all performed without 3D CT characterization. Therefore, this work proposes a general setup that combines all of the above-mentioned qualities.

Let us examine the existing in-situ devices in the literature in more detail. Poulet et al. [3] investigated the void initiation and evolution of a semi-crystalline polymer by in-situ Synchrotron experiments, using a device that was limited to tensile loading and specific sample geometries. Buffiere et al. [4] proposed a testing design that enables both in-situ CT tensile and compression experiments, however, the authors noted a reduction in CT resolution due a large source-sample distance and off-centric rotation of the sample during CT scanning, both caused by the chosen size and location of the stepper motor. Buffiere et al. [4] and Gueninchault et al. [5] solved these two problems, by designing an in-situ $\mathrm{CT}$ tension/compression device in which all heavy (large) parts were located at the bottom of the device, enabling tensile and compression experiments under high resolution CT scans [15].

Parallel to the development of these in-situ mechanical loading devices, (hygro-)thermo conditioning devices with and without mechanical loading have been proposed. Bellet et al. [6] were the first to design a furnace for in-situ CT heating, while various similar devices are now commercially available. Obvious interest exists in combining these loading and conditioning capabilities. For instance, the mechanical loading device of Buffiere et al. [4], addressed above, also includes induction heating up to $1500{ }^{\circ} \mathrm{C}$, which is only suited for conducting samples. Bertrand et al. [7] created their own thermomechanical loading device and used it to test the selfhealing properties of carbon-coated $\mathrm{SiC}$ fibre composites, by alternating mechanical loading and conduction heating to $800{ }^{\circ} \mathrm{C}$. Finally, Case et al. [8] developed, for in-situ X-ray diffraction analysis, a compact combined relative humidity - temperature chamber, to investigate the phase behaviour of aligned DNA fibres as a function of hydration, in which they added a so-called 'DNA strand puller' that was designed specifically for very delicate samples, whereas no force measurement was realized; more importantly, the setup is only transparent to X-rays at limited rotation angles, which is sufficient for X-ray diffraction but not for X-ray CT.

For a different class of problems, concerning large deformation and localization phenomena in combination with a required high resolution and thus small samplesource distance, a key problem is that the sample's region of interest (ROI) moves out of the detector's field of view (FOV) when loading from only one side. Maksimcuka et al. [9] found a partial solution to this problem, in which the sample was still loaded from one side, resulting in a non-stationary ROI while a compensating upwards motion of the whole tensile system moved the ROI back in the FOV, thereby achieving a voxel size of $0.81 \mu \mathrm{m}$ at $352 \%$ strain. A more elegant solution was proposed by Chen et al. [10], who designed a system for in-situ Synchrotron CT scanning, which consists of two dual rotation-loading actuators to symmetrically load the sample, resulting in a stationary ROI, although at the expense of larger setup dimensions that are incompatible with most lab-scale CT devices.

The aim of this paper is to develop, validate and test a general in-situ lab-scale CT deformation device, which overcomes the above-mentioned limitations of existing setups. To this end, a requirement list has been formulated, which, if met, enables the above-mentioned hygro-thermo-mechanical test (on paper, CFRP, foam, biological specimens, etc.) under in-situ CT imaging with precise control and measurement resolution of the load, displacement and environmental conditions. This requirement list is based on the following considerations:

First of all, the device should enable material testing under various loading conditions, i.e. tensile, compression, torsion, bending. Additionally, investigation of timedependent material behaviour, such as creep and relaxation, should be possible, requiring, respectively, force and displacement controlled experiments. Finally, a modular sample clamping principle is required for fixing rectangular and circular (less CT scanning artefacts) samples and specific loading modules which allow for, e.g., three- and four-point bending tests.

Next, exchangeable loadcells and an extensiometer are required to allow precise material characterization. Ideally, the setup should be able to deform a wide range of samples to failure in order to study the corresponding failure mechanisms (delamination, buckling, fracture). A survey of potential material of interest for in-situ CT hygro-thermo mechanical testing (carbon-fibre reinforced polymers, foams, paper/cardboard), considering their materials properties, fracture strain and typical sample geometries, revealed the following, minimum, criteria for the applicable load and displacement range: $2 \mathrm{kN}$ and $5 \mathrm{~mm}$.

Additionally, the setup should allow high resolution CT characterizations with the minimum voxel size specified by the particular CT scanner, hence minimization of the source-sample distance is required. While, off-centric rotation should also be minimized to reduce the amount of scanning artefacts. To this end, the weight of the setup should be evenly distributed over the air bearing, which requires a rotational symmetric design. If done properly, the deterioration of the $\mathrm{CT}$ resolution should be negligible. Note that, the inevitable X-ray transparent sample tube to transfer the load across the sample will always deteriorate the CT resolution, however further deterioration by the setup weight and unbalance can be avoided. Loading 
the sample equally from both sides and hence having a stationary ROI is also an important requirement as it enables automatic multiple-step long-duration experiments. Finally, mechanically testing samples under severe processing and transport conditions $\left(10^{\circ} \mathrm{C}<\mathrm{T}<60{ }^{\circ} \mathrm{C}\right.$ and $10 \%<\mathrm{RH}$ $<90 \%$ ) requires an enclosed volume around the sample in which the climate is controllable.

In short, the above-mentioned considerations lead to the following design requirements:

- Deformation using multiple loading modes:

- Conventional tension and compression

- Creep and relaxation by load- or displacement control

- Different boundary conditions, e.g., three- and four-point bending

- A modular clamping method for circular and rectangular samples

- Preservation of sub-micrometer scale CT resolution

- The same CT resolution for a sample with sample tube with or without the setup.

- ROI centre stationary within $10 \mu \mathrm{m}$

- Force and displacement accuracy and range

- Load range up to $2 \mathrm{kN}$

- Force measurement resolution of $0.01 \mathrm{~N}$

- Travel range up to $5 \mathrm{~mm}$

- Displacement measurement resolution of $1 \mu \mathrm{m}$.

- Climate control at severe transport and processing conditions

- Temperature range of 10 to $60{ }^{\circ} \mathrm{C}$.

- Relative humidities range of 10 to $90 \%$.

In the following, first, the conceptual design is introduced and worked out in detail (Conceptual Design and Realisation), followed by a validation of the abovementioned setup requirements (Setup Validation), after which proof-of-principle hygro-thermo-mechanical experiments on cardboard are presented (Proof of Principle Experiment: Creasing and Folding of Cardboard). Finally, conclusions are drawn and recommendations for future extensions are discussed.

\section{Conceptual Design and Realisation}

The design space for in-situ CT devices is limited due to the hardware inside the CT scanner (i.e. CCD Detector, $\mathrm{X}$-ray source, position actuators). Therefore the hereproposed device is designed for sub-micrometer resolution
CT scanners that have a small design space [16-19]. Particularly, the design space of the Nanotom $160 \mathrm{NF}$ by General Electric/Phoenix $\left(\oslash 140 \times 205 \mathrm{~mm}^{2}\right)$ is set as a requirement as this $\mathrm{CT}$ scanner has the smallest design space. The specific space constraints are as indicated in Fig. 1: (a) a source-rotation-stage distance of $130 \mathrm{~mm},(b)$ a sample's centre height of $205 \mathrm{~mm},(c)$ a maximum allowable radius of $70 \mathrm{~mm}$ and $(d)$ a maximum source-sample distance of $10 \mathrm{~mm}$. Additionally, this CT scanner puts the highest constraint on the weight of the in-situ device, i.e. only $2.0 \mathrm{~kg}$ is allowed.

After extensive deliberation of various options, the following conceptual design is proposed that complies with all of the above-mentioned constraints and requirements, see Fig. 1. In the conceptual design the upper and lower sample clamp are rigidly attached to two elements (coloured, respectively, red and orange) which must slide neatly over each other in vertical direction, both elements having an equal but opposite motion, resulting in a stationary ROI. This motion is driven by precision vertical linear actuators that are placed at the bottom, allowing them to slide under the X-ray source. Note that the actuator's choice is thus greatly influenced by the sourcerotation-stage distance (a). In this configuration, the sourcesample distance (d) is only limited by the sample tube's radius.

In addition to ensuring precise motion, the applied force and displacement are accurately monitored using, respectively, a loadcell and three extensiometers (LVDT's), while the LVDT's provide feedback on the axial alignment. The loadcell is exchangeable allowing accurate force measurement over a large force range, for various types of specimens. Additionally, in the enclosed environmental chamber around the sample (purple), the climate is controlled using a humidity/temperature sensor near the sample and an air circulation system with in- and outlet from the top. At last, the setup is fixed on the precision rotation stage that is always available in a CT system. The proposed design should not exceed the weight limit of the air bearing inside the rotation stage, as this causes offcentric rotation to occur. Moreover a rotational symmetric design is chosen to evenly distribute the weight over the air bearing.

The proposed conceptual design is subsequently realised into a detailed design of the in-situ CT tester, shown in Fig. 2, of which the specific design considerations are discussed next. The main components are highlighted and the colours (see legend) indicate the motion or the function of the different components. A section view (a) gives an overview of the location of the main components.

Let us start with the actuation of the proposed setup. The biggest specific challenge here is actuating the device 


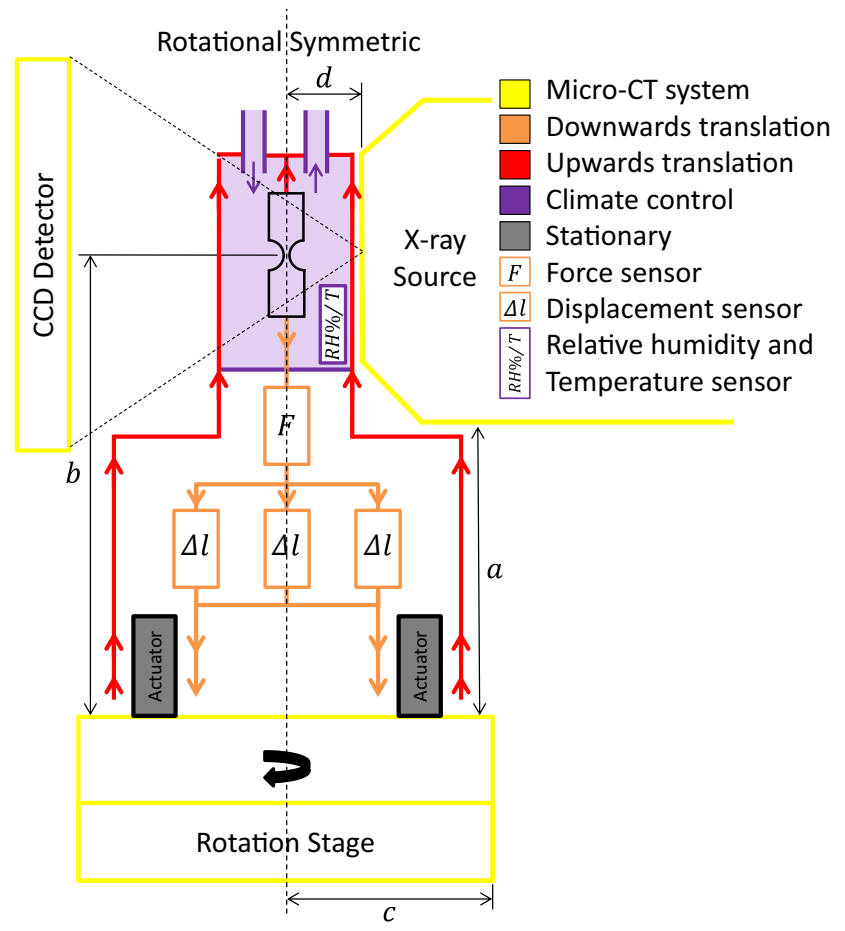

Fig. 1 Schematic representation of the conceptual design. The space constraints corresponding to the small design space of the Nanotom 160NF CT scanner are: $a \leq 130, b \leq 205, c \leq 70 \mathrm{~mm}$ and $d \leq 10 \mathrm{~mm}$. The legend indicates the type of motion or its functions of the different components. The inside element (orange), with force and displacement sensors, makes a downwards motion while the outside element (red) makes an equal upwards motion, resulting in a tensile actuation with a stationary ROI. In the enclosed area (purple) the temperature and humidity is controlled

with both up- and downwards translation, high applied load, small precise steps, sufficient stroke and enough space for sensors all within the compact design space $\left(a \pi c^{2}\right)$ of the CT scanner. A comprehensive analysis of possible actuator choices was performed. For instance, Buffiere et al. [4] and all of the commercially available devices [20] use a single electro motor (with gearbox) in combination with a lead screw to generate a linear motion. However, this requires dual linear actuators for equal up- and downwards translation. Gueninchault et al. [5] used a specifically designed piezoelectric actuator which could apply forces up to $450 \mathrm{~N}$ and a maximum stroke of $500 \mu \mathrm{m}$, however, this also requires dual linear actuators to attain equal up- and downwards translation and the applied force and stroke are limited. Equal up- and downwards translation with sufficient applied force and stroke was attained by Chen et al. [10], however, their design does not fit the tight space constraints of the chosen CT system. Therefore, none of these options are applicable for the here-proposed setup. Rotation (servo) motors have potentially better specifications than linear actuators, however, only when the rotational motion can effectively be converted into linear actuation. Hence the following key design choice for vertical double actuation was made: A planetary gear $(b)$ is used to convert the rotational motion of three servo motors in both an up- and downwards actuation by means of, respectively, an (outer) ring gear and an oppositely rotating (inner) sun gear.

The planet gears, which drive the ring and sun gear, can only rotate and are individually driven by a servo motor. Dimensions are chosen such that gear ratios of 1:14 (sun : planet) and 1:16 (ring : planet) are realised, allowing easy gear ratio difference compensation by the subsequent transmission in order to achieve equal upwards and downwards translation of the top and bottom clamp. A Keramo finish surface treatment reduces the surface roughness of the electrical discharge machined gears. The sun- and ring gear are both connected to a threaded cylinder $(c)$. When driven, the ring gear rotates in counter-clockwise direction (green) and the sun gear in clockwise direction (blue), resulting in, respectively, an upwards translation (red) of the upper plate and a downwards translation (orange) of the lower plate, see Fig. 2(c). A stationary ROI is required, hence the difference in rotational speed between sun- and ring gear is compensated by the pitch of the two cylinders, respectively, $1.4 \mathrm{~mm} / \mathrm{rot}$ and $1.6 \mathrm{~mm} / \mathrm{rot}$. A spline $(d)$ is used to block the lower plate from rotation, which underwent the same surface treatment as the planetary gear. Three linear bearings $(e)$ are used to block the upper and lower plate from rotation with respect to each other, thereby realizing a precise linear vertical motion without backlash. However, these bearings do over-constrain the system, which requires precise machining of the connected parts to reduce internal forces. To extend the precise linear motion towards precise vertical clamp displacement, an additional radial bearing $(e)$ has been inserted just below the bottom clamp. An important feature of this configuration is that both the ring- and sun gear are loaded during a test, resulting in low lateral forces on the motor shafts even for loads up to $2 \mathrm{kN}$. Translation of the upper and lower plate is blocked by the screw thread, which is ideal for relaxation experiments. Relaxation tests are realised by driving to the set-point and subsequently stopping the motors, because the displacement can be assumed constant due to sufficiently high friction in the threads $(c)$. For creep tests, the force from the loadcell is used as input for a feedback control loop of the applied displacement.

These design choices made for precise actuation leaves a limited space for the data acquisition. Hence, measuring force and displacement and additionally receiving feedback on axial alignment all within a compact design space of $\oslash$ $43 \times 80 \mathrm{~mm}^{3}$ is a challenge. In most of previously discussed setups the loadcell is placed in line with the loading direction to attain accurate force measurements. Here too, the loadcell $(f)$ is placed axis-centric in line with the loading direction. For accurate force measurements, minimization 


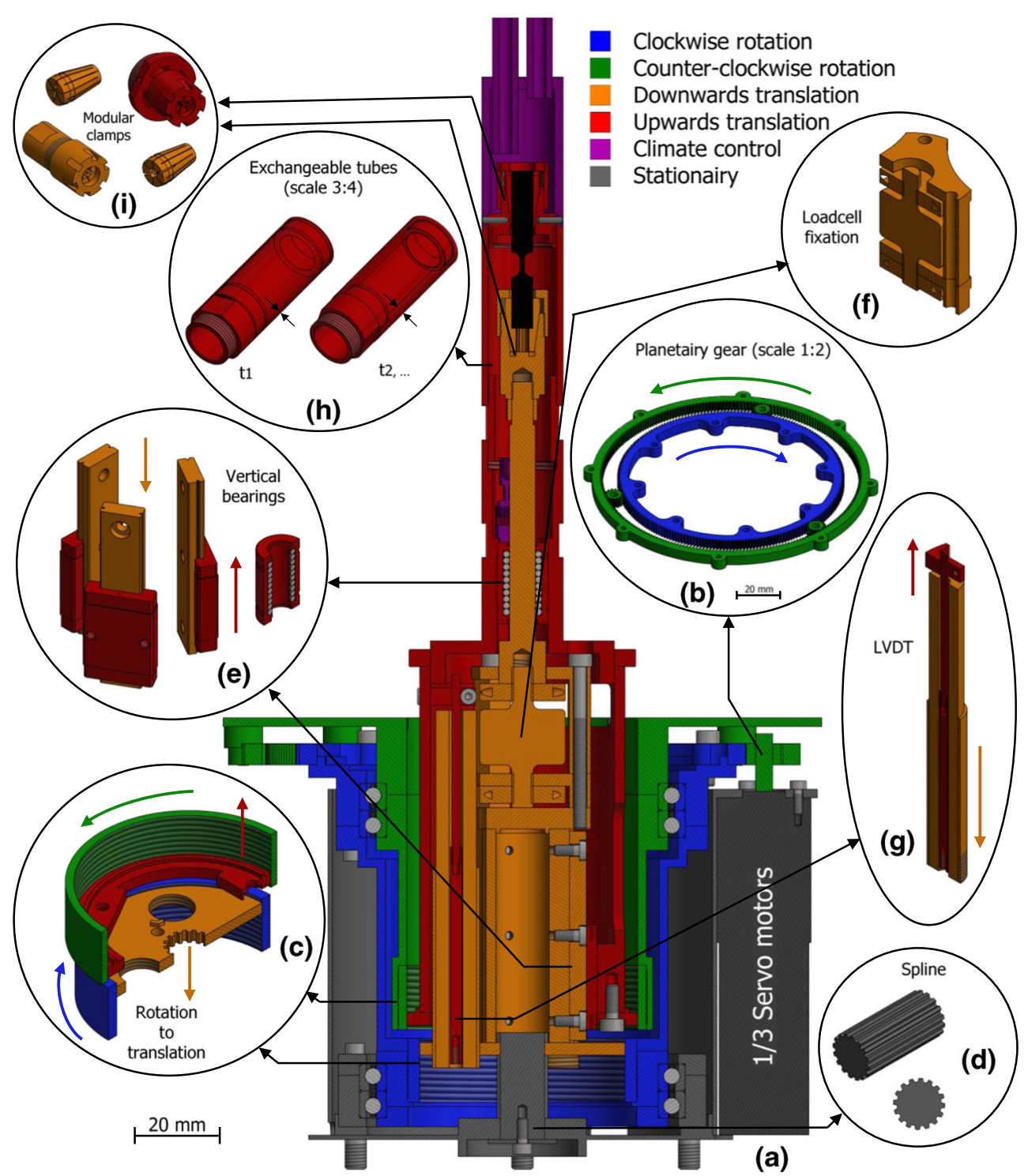

Fig. 2 A CAD model of the detailed design of the in-situ CT tester. (a) section view of the realised setup, $(b)$ planetary gear which is driven by three servo motors, $(c)$ two large threaded cylinders (green and blue) which convert rotational motion of the planetary gear into vertical translational motion of the upper (red) and lower (orange) plate which are connected to the sample clamps, $(d)$ spline that is fixed to the base which blocks rotation of the inner cylinder, $(e)$ vertical bearings which block the outer cylinder from rotation with respect to the inner cylinder, thereby enforcing a precise linear translation, $(f)$ loadcell which is fixed under the clamp for accurate force measurement, $(g)$ three LVDT's which accurately monitor the relative displacements of the cylinders, $(h)$ exchangeable tubes and $(i)$ universal clamps which are easily exchangeable. Note the legend, i.e. each colour indicates a different motion

of off-axial loading of the loadcell is imperative, hence two counter-nuts are used to reduce backlash within the loadcell's threads, as is also done in [21]. This design also allows the loadcells to be exchanged.

For most of these setups the displacement is retrieved from the motor encoder steps. However, this method does not take into account the setup's compliance, hence, a different design using three LVDT's (one MHR250 and two MHR500 LVDT from Measurement Specialities) is employed to achieve accurate displacement measurements and compliance calibration. The setup has a maximum stroke of $24 \mathrm{~mm}$, however to avoid collision within the system, a safety range from 0 to $20 \mathrm{~mm}$ is chosen. The three LVDT's $(g)$ are used to measure this stroke: one covers the first $12 \mathrm{~mm}$ with a resolution of $1 \mu \mathrm{m}$, while the remaining two cover the full stroke with a resolution of $2 \mu \mathrm{m}$. A constant check is performed to see if the current displacement is within the given safety range. In addition, Digital Image correlation (DIC) on a 2D X-ray image of the complete sample ROI [22] or the sample's notch 
opening [3] can be used to attain, respectively, accurate local displacement or strain measurements directly of the sample. However these techniques are cumbersome, not real time, do not provide feedback on axial alignment and requires enough X-ray contrast for proper DIC correlation.

The sample tube $(h)$ must be made of a highly X-ray transparent amorphous material, e.g. polymethylmethacrylate (PMMA), which is important for avoiding unwanted diffraction of X-rays. The tubes are easily exchangeable and can have a maximum thickness of $3 \mathrm{~mm}$. The thickness is chosen based on the sample's absorption coefficient and the required stiffness to carry the maximum load. Materials such as glassy-carbon [20] or quartz [5] can be used instead of PMMA if a stiffer tube is desired. For the design samplesource distance of $d=10 \mathrm{~mm}$ (Fig. 1), the thinnest sample tube is selected which has an outside radius of $9.5 \mathrm{~mm}$ and, together with a maximum tube thickness of $3 \mathrm{~mm}$, the maximum scan-able sample size is limited to $\oslash 12.5 \times 30 \mathrm{~mm}$. This radius can be increased, by replacing the extension tube by one with a larger radius, however, at the expense of a lower resolution.

Being able to clamp, respectively, rectangular and circular samples with minimal sample geometry adjustments requires a modular clamping technique. Maksimcuka et al. [9] drilled holes in their sample and used fasteners to fixate them, which inevitably induces high stress localizations around the hole. Reinforcement by means of glueing tabs is required for this clamping technique when applying it to thin sheet material [23], which is unideal. Poulet et al. [3] proposed a specimen that avoids unwanted stress localizations around drilled holes, however, the resulting range of allowable sample geometries is too specific for our purpose, while these samples can only be loaded in tension. Instead, the clamping principle of a collet drill head would suffice for the proposed setup [24]. However, downscaling and adjusting the collet geometry is required to allow fixation of rectangular and circular samples within the small design space of the sample tube with an inner radius of $6.5 \mathrm{~mm}$. Additionally, no brute clamps are required since the maximum applied load is relatively low. Hence, the design choices made for the here-proposed setup resulted in two modular clamps $(i)$, which uses a commercially available milling collet to either clamp cylindrical samples and micro-machined milling collets to clamp rectangular samples. They are easily exchangeable, allowing the user to clamp cylindrical samples with maximum diameter of $5 \mathrm{~mm}$ or rectangular samples with a maximum width and thickness of, respectively, 5 and $1 \mathrm{~mm}$.

Allowing hygro-thermal-mechanical testing requires a controlled climate inside the sample tube. The specific challenge in realizing a climate-controlled environment is attaining high enough temperature and relative humidity range within acceptable error bounds, which requires sufficient isolation to minimize any losses. Betrand et al. [7] and Buffiere et al. [4] used a copper induction coil to heat up the sample, however, this is not applicable for isolators and biological materials. It would suffice to minimize the design proposed by Case et al. [8], in which the temperature and relative humidity from the sensors are used as an input for a feedback control loop for the heating elements. However, miniaturization of Case's design to fit the space constraints is unrealistic. Instead, in the hereproposed design an external (large-scale) climate box [11] is connected with flexible tubing to an in- and outlet at the top of the environmental chamber around the sample. This climate box uses feedback control from a relative humidity and temperature sensor near the sample to maintain the set relative humidity and temperature. Note that the upper clamp has an open structure, allowing the humidity and temperature controlled air to flow towards the sample. For proper temperature control, an additional heating/cooling element is fixed around the flexible in- and outlet tubing, right above the setup within the CT chamber. Two flexible well-isolated hoses allow airflow from this element to the setup.

A CAD model $(a)$ and photograph $(b)$ of the realized setup, mounted on the precision rotation stage, are shown in Fig. 3.

The electrical control box $(c)$ is placed next to the source, in close vicinity of the setup, thereby allowing short cables. The precision rotation stage applies a relatively low torque to induce the rotation for CT scanning. Therefore, a significant challenge lies in choosing the right cable types and exit locations to minimize the forces and moments that the cables exert on the setup, which would induce a detrimental effect on the rotation. In the proposed design, room is created to allow the wires from the LVDT $(d)$, loadcell (e) and one of the temperature/relative humidity sensors to exit the setup from the bottom, through the centre of the precise rotation stage, after which they directly go to the control box. By pre-twisting these wires, the torque needed for rotation becomes negligible. The servo motors are mechanically coupled to each other, hence only one encoder is wired to the control box, which allows a single cable with less channels. This cable cannot exit the setup through the bottom centre because the motors are located outside the rotating and vertically translating cylinders and thus needs to exit from the side of the setup. To this end, a flat flex cable $(f)$ is used, which is wound around the setup, which allows the setup to make the full $360^{\circ}$ rotation. The in- and outlet hoses for climate control $(g)$ and the second temperature/relative humidity sensor cable exits from the top centre. These are wired via the ceiling of the CT scanner chamber to, respectively, the climate box and PC, and again pre-twisted. Finally, a LabVIEW control program has been written to enable multi-step autonomous experiments. 

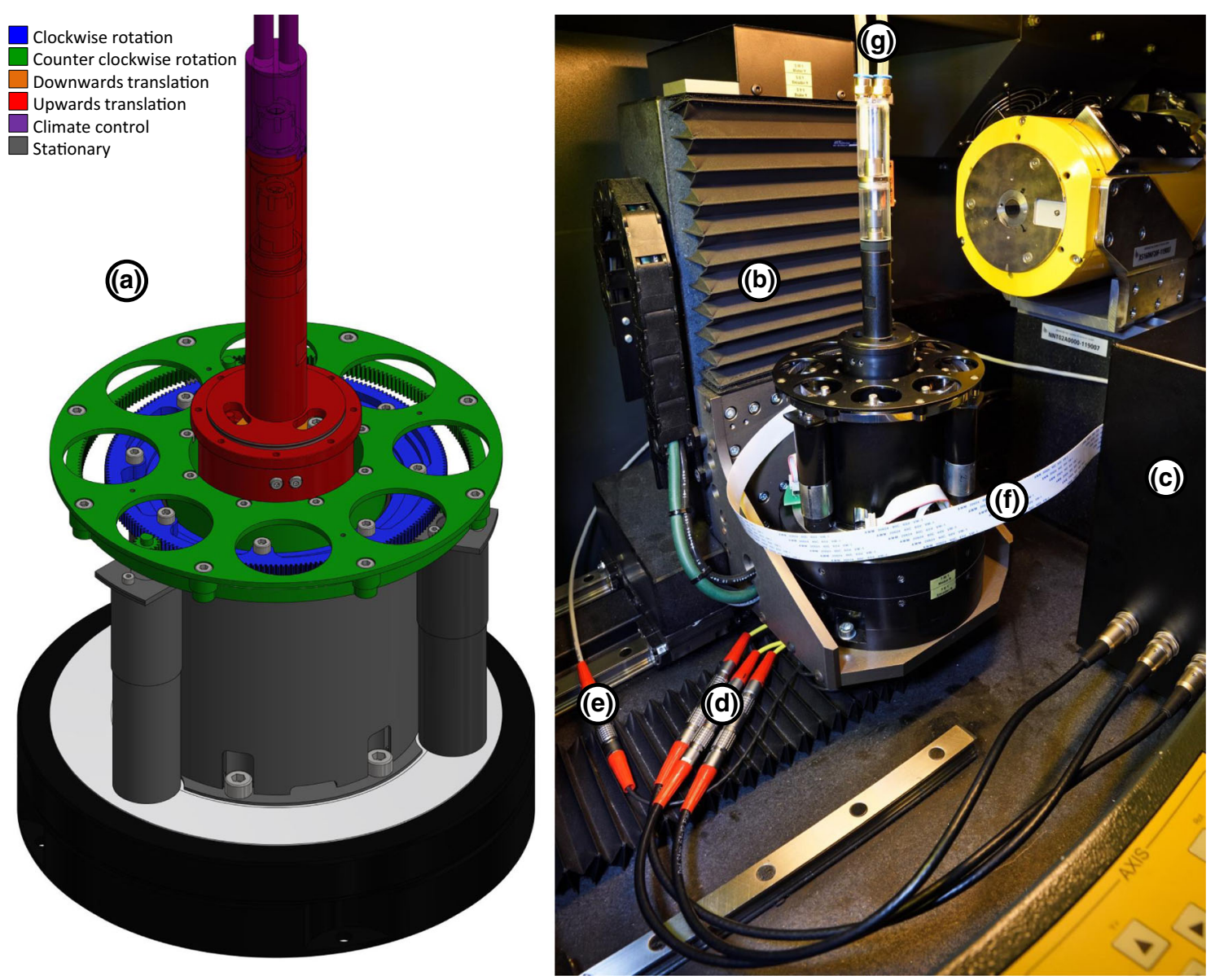

Fig. 3 Overview of the realized in-situ CT tester mounted on the precision rotation stage: $(a)$ the CAD model, $(b)$ the realistic setup, $(c)$ control box with wiring sockets, $(d)$ LVDT wires, $(e)$ loadcell wire exiting from the setup bottom, $(f)$ flat flex wire, wound around the setup to enable Xray scan rotation, to drive the motors and read the encoder and $(g)$ in- and outlet of the climate box along with the cable of a combined temperature and relative humidity sensors. A total of two temperature and relative humidity sensors are present, one above and one below the sample

\section{Setup Validation}

Multiple validations experiments are conducted to verify that the above mentioned requirements are met by the proposed device.

First, the CT reconstruction quality is validated by doing three CT scans of the same aluminium foam sample, i.e. without sample tube, with sample tube and in the realised setup (with sample tube and additional weight). This allows analysis on the possible detrimental influences of a sample tube and possible off-centric rotation due device weight unbalance on the image reconstruction. A PMMA sample tube with outside radius and thickness of, respectively, 9.5 and $0.5 \mathrm{~mm}$ is used for the last two mentioned situations. The 2D X-ray images, for all three configurations, were collected with a voltage of $60 \mathrm{kV}$ and a current of $300 \mu \mathrm{A}$. A total of 1400 images with an exposure time of $500 \mathrm{~ms}$ and an average of 6 were collected. An effective voxel size of $4 \mu \mathrm{m}$ was attained to properly capture the aluminium surface roughness features. The 3D-characterization are given in Fig. 4.

To allow a more convenient comparison and analysis, a zoomed in view of a featured area is provided. A distinct loss in detail, due to X-ray attenuation by the sample tube, is visible when comparing image $a$ with images $b$ and $c$ (note that the 3D rendering lighting conditions are different for each scan due to the different initial sample rotation). Due to space constraints, usage of a sample tube is inevitable for this class of lab-scale in-situ CT test devices, see [3-10]. However, the surface roughness and other small features such as voids are still clearly characterized and distinguishable with sample tube. Comparing images $b$ and $c$ allows analysis on the effect of a possible weight unbalance of the proposed setup on the 3D reconstruction quality. Both reconstructions are highly similar and no additional scanning artefacts could be observed in image $c$ due to the possible off-centric rotation. Additionally, a quantitative resolution analysis has been performed on 

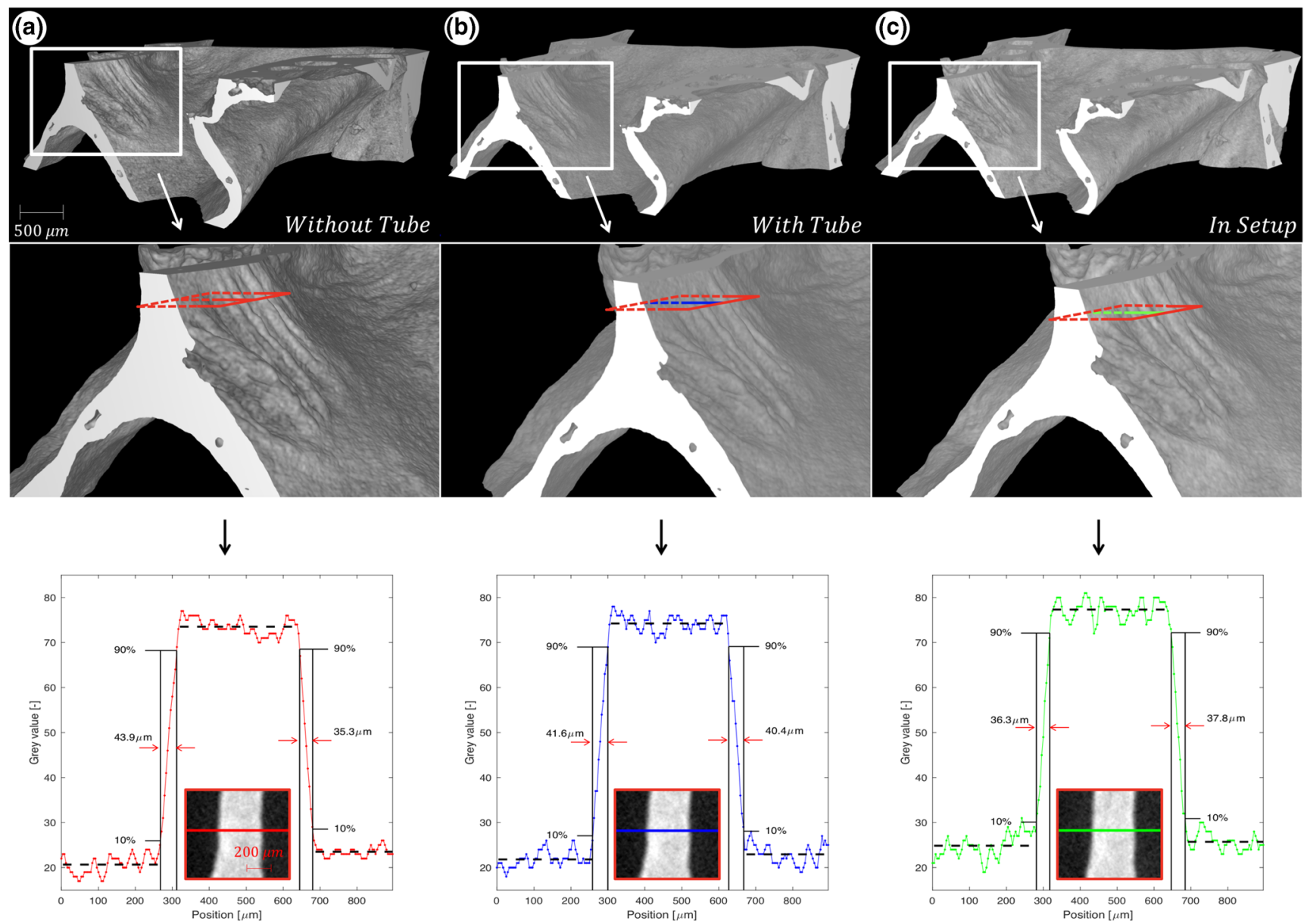

Fig. 4 3D-characterisation of aluminium foam scanned with: $(a)$ without sample tube, $(b)$ with sample tube and $(c)$ inside the realised device. A zoomed in view is provided. Note that the $3 \mathrm{D}$ rendering lighting conditions are different for each scan due to the different initial sample rotation. At the bottom three 2D images of the same sharp aluminium edge are provided, on which a quantitative analysis has been performed along the red, blue and green line to determine the CT resolution, which is approximately $40 \mu \mathrm{m}$ in all three cases

three 2D images of the same sharp aluminium edge within the samples, see bottom of Fig. 4. The CT resolution is evaluated by measuring the distance of the grey value profile (along, respectively, the red, blue or green line) as it changes from 10 to $90 \%$ and from 90 to $10 \%$ at the sharp aluminium edge. The resulting resolution values (of approximately $40 \mu \mathrm{m})$ are indicated in the Figure and are equal within experimental uncertainties for the three cases, hence no CT resolution reduction is present.

Second, to ensure a stationary ROI, two shafts are clamped by, respectively, the top and bottom clamp. 2D CT images with an effective pixel size of $1.5 \mu \mathrm{m}$ are used to trace two pixel locations on the edge of the shaft (red and green in Fig. 5) during seven increments of each $100 \mu \mathrm{m}$. After each increment, the pixel location is determined by the correct grey value. The absolute displacement difference between the two shafts is plotted in Fig. 5 and stays within one pixel variation, corresponding to $1.5 \mu \mathrm{m}$. This is well below the required $10 \mu \mathrm{m}$ limit, which demonstrates the precise dual linear actuation of the novel planetary gear driving mechanism.

Third, to ensure accurate load an displacement measurements, a validation is performed by measurement of the known Young's modulus of brass $\left(\mathrm{CuZn}_{39} P b_{3}\right)$ by performing tensile tests on three cylindrical brass specimens with a radius of $1.5 \mathrm{~mm}$. To this end, first, the machine compliance (loadcell, tube and system deformation) calibration curve is attained by loading and unloading a steel shaft with a radius of $2.5 \mathrm{~mm}$ to $1 \mathrm{kN}$. The force displacement relationship is presented in Fig. 6(a). A linear fit is made which is used for machine compliance correction of the measured displacement to obtain the real clamp displacement.

Subsequently, this linear calibration curve is subtracted from the measured displacement data of the three brass 


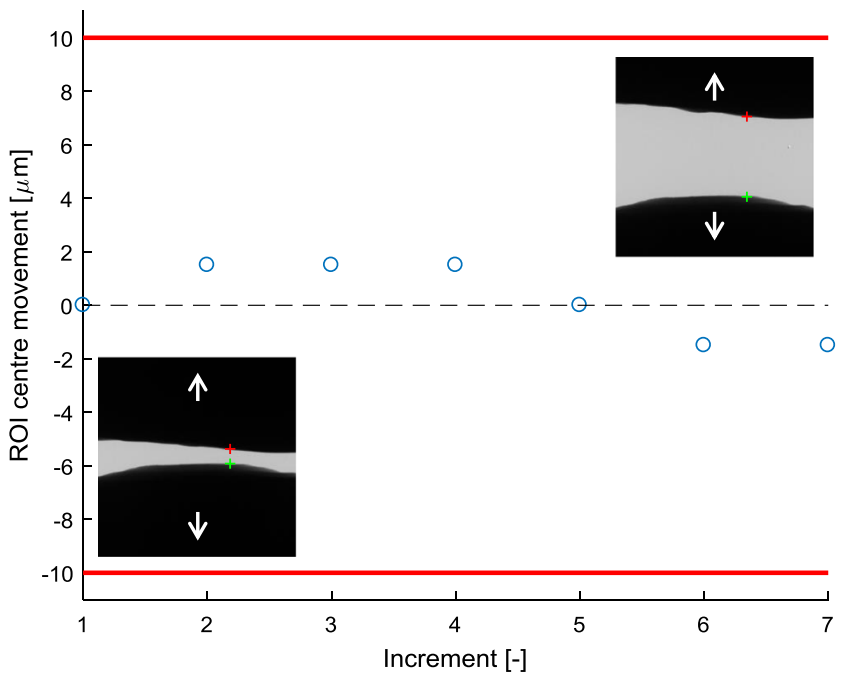

Fig. 5 Stationary ROI validation, two points (red and green) are tracked during seven displacement increments of $100 \mu \mathrm{m}$ and used to determine the ROI centre movement. This is plotted and stays well within the required limit (red lines)

tensile tests. The corrected stress-strain curves in the unloading regime are given in Fig. 6(b). For clarity, a fivetimes averaging is applied to the displacement data and an artificial offset in strain is given to each of the three tests. Each sample shows a similar response which demonstrates reproducibility of the load and displacement measurements. The average Young's modulus of the tested samples is then determined and equals $99.7 \pm 5.4 \mathrm{GPa}$, which is in good agreement with the specified modulus of $97 \mathrm{GPa}$ of the tested $C u Z n_{39} P b_{3}$ alloy. At last, it is noted that the setup allows for exchangeable loadcells and the loadcell fixation design is generic, allowing most commercial loadcells.

Fig. 6 Loadcell and extensiometer validation with: (a) machine compliance calibration curve obtained from both loading (blue) and unloading (red) as steel shaft with radius of $2.5 \mathrm{~mm}$, fitted with a first order polynomial (black) and (b) Young's modulus determination of brass samples with a radius of $1.5 \mathrm{~mm}$, showing linear elastic unloading curves with correct Young's modulus $(E=99.7 \pm 5.4 \mathrm{GPa})$, after machine compliance correction
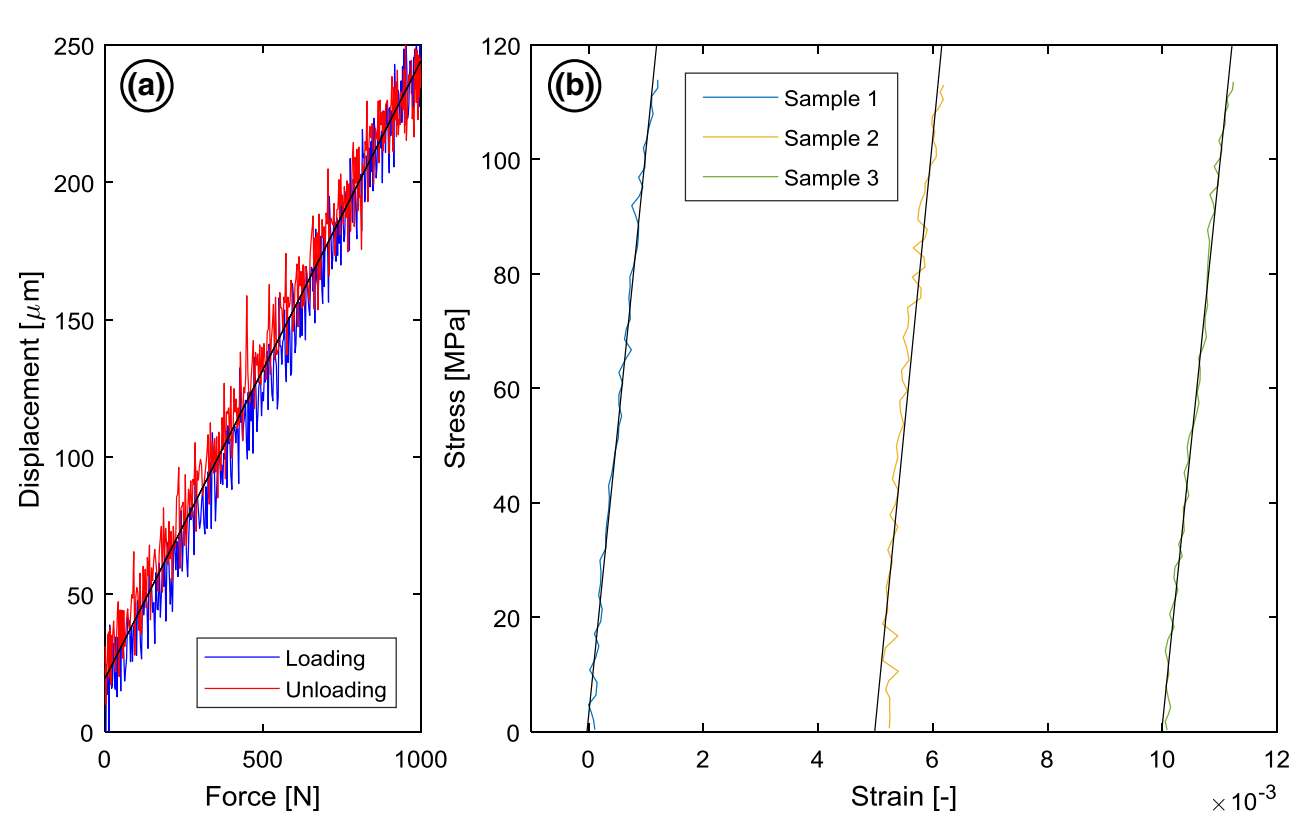

At last, to ensure proper climate control, various temperatures and relative humidities are tested for $1300 \mathrm{~s}$ and shown in Fig. 7. The above-mentioned temperature and relative humidity range requirements are shown as red dashed lines. Temperatures ranging from 10 to $75^{\circ} \mathrm{C}$ are realized by the device, which exceeds the abovementioned requirement. Minor fluctuations are visible at higher temperatures which originate from the limited sample tube isolation, which is inevitable. Additionally, a relative humidity range of 10 to $99 \%$ is realized, which also exceeds the above-mentioned requirement.

\section{Proof of Principle Experiment: Creasing and Folding of Cardboard}

The setup's potential is demonstrated by performing a complex hygro-thermo-mechanical experiment in which consists of (i) creasing, (ii) folding and (iii) climate control of a multi-layer cardboard in a climate-controlled environment. The required bending moment for folding cardboard greatly decreases when it is priorly creased [25]. These creasing and folding experiments on multilayer cardboard were previously performed on a larger samples by Beex et al. [12]. By miniaturizing their loading modalities to fit inside the here-proposed in-situ CT device, full 3D CT characterization of the cardboard during these complex experiments becomes possible. Due to its interesting intrinsic behaviour during the creasing and folding process, multi-layer cardboard is used here to demonstrate the setup potential, where it is noted that the $\mathrm{X}$-ray absorption coefficient of paper fibres is low and similar to that of the PMMA tube making this test highly challenging. 

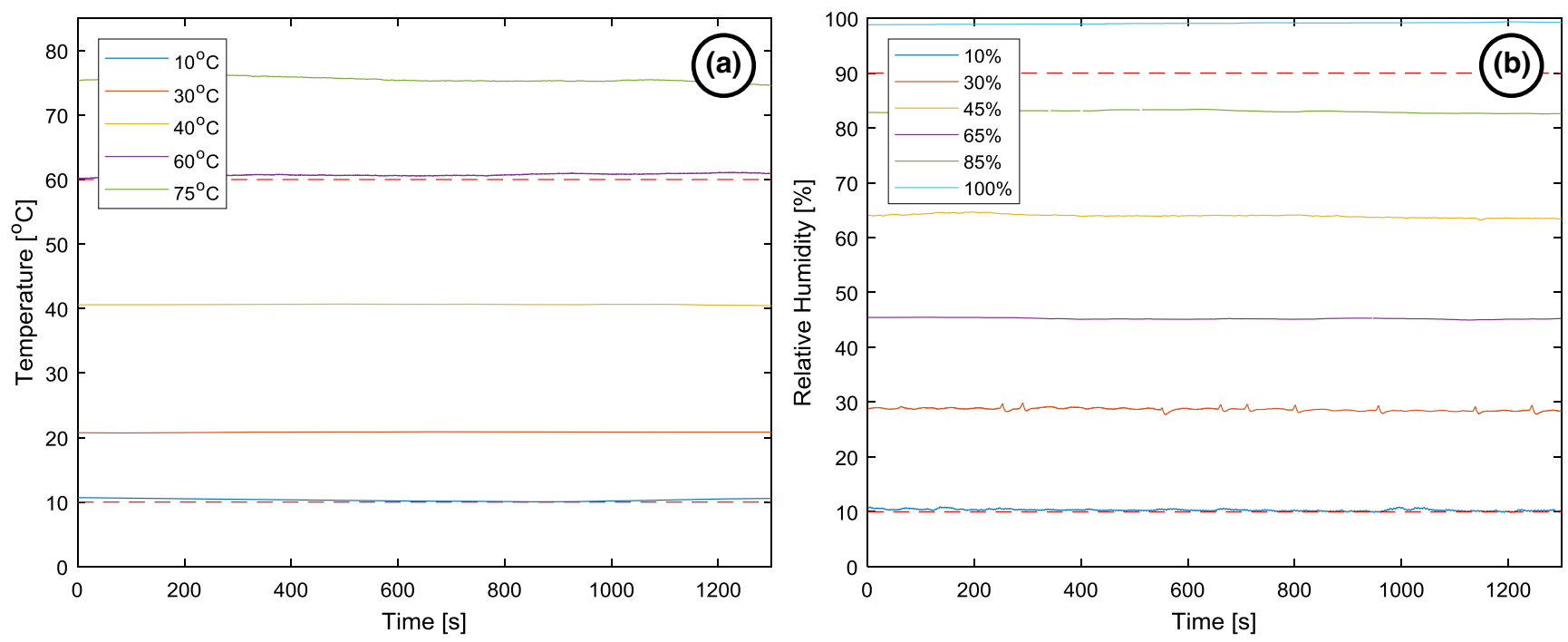

Fig. 7 Climate-control validation plots with: (a) temperature-controlled environment and $(b)$ relative humidity-controlled environment. The red dashed lines indicate required temperature and relative humidity range

\section{Creasing and Folding}

A schematic presentation of the experimental three- and four-point bending modules that were used by Beex et al. to study the creasing and folding process is shown in Fig. 8(a). Their loading module had been miniaturized to fit inside the here-proposed in-situ CT device. The creasing channel has a width and height of, respectively, 2.50 and $1.00 \mathrm{~mm}$, the creasing rule has a radius of $0.525 \mathrm{~mm}$ and the crease depth is $1200 \mu \mathrm{m}$. The supports' radius in the four-point bending module is $0.5 \mathrm{~mm}$ while the centres of the supports have a distance of, respectively, 16 and $6 \mathrm{~mm}$. These dimensions are equal to the values used in [12]. Figure $8(b)$ shows two light microscopy images taken earlier during the creasing and folding process [12]. Delamination of the different layers is clearly visible after folding the sample.

Mayr-Melnhof Eerbeek provided $840 \mu \mathrm{m}$ thick cardboard samples consist of three layers: a $50 \mu \mathrm{m}$ pigmented bottom-layer, a $740 \mu \mathrm{m}$ mid-layer and a $50 \mu \mathrm{m}$ shiny double pigmented top-layer. The mid-layer itself is divided into one inner and two outer layers, which consist of, respectively, mechanical pulp and bleached chemical pulp from a mixture of virgin fibre (90\%) and pigmentation (10\%) material. The width of the sample parallel to the creasing channel is $4 \mathrm{~mm}$ and the length perpendicular is $12 \mathrm{~mm}$. The threeand four-point bending modules have been machined from

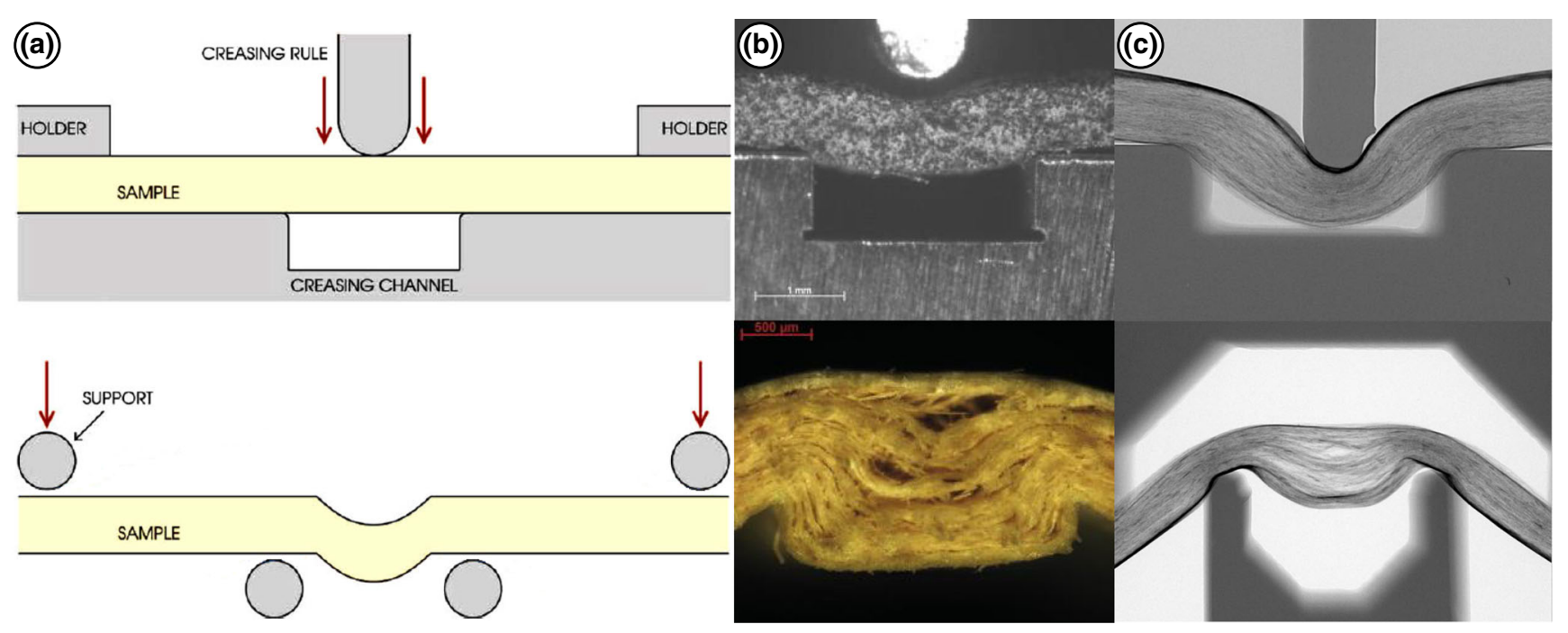

Fig. 8 Experimental setup with: (a) Schematic representation of the three-(top) and four-point bending (bottom) modules [12], (b) The earlier light microscopy images [12] and (c) new 2D X-ray images taken during the creasing (top) and folding experiments (bottom) 


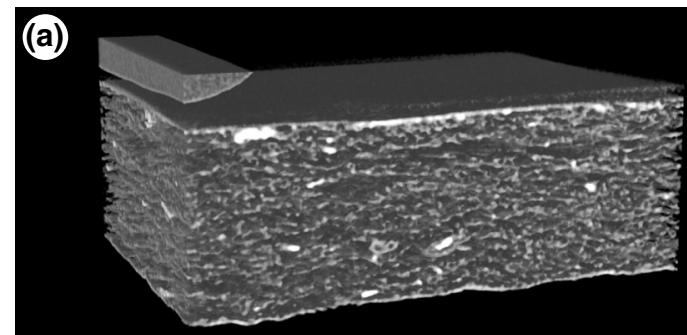

(c)

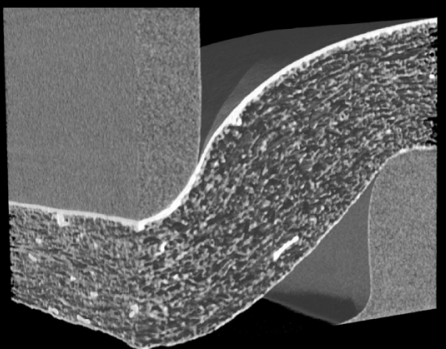

(b)

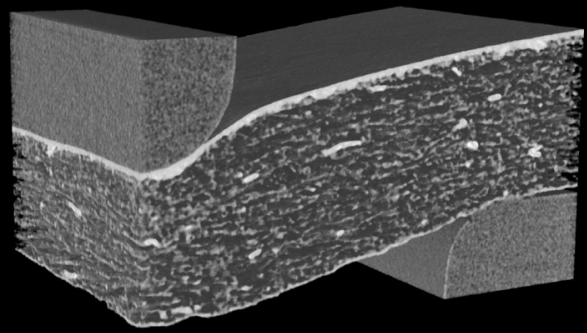

(d)

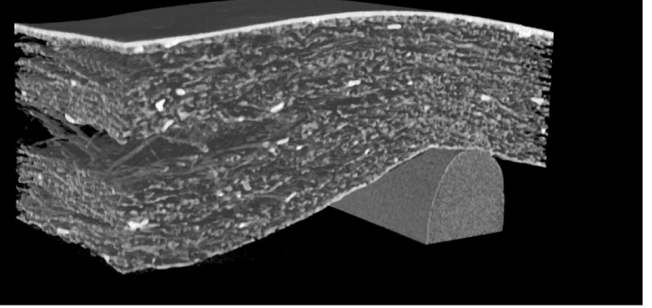

$4^{\times 10^{4}}$
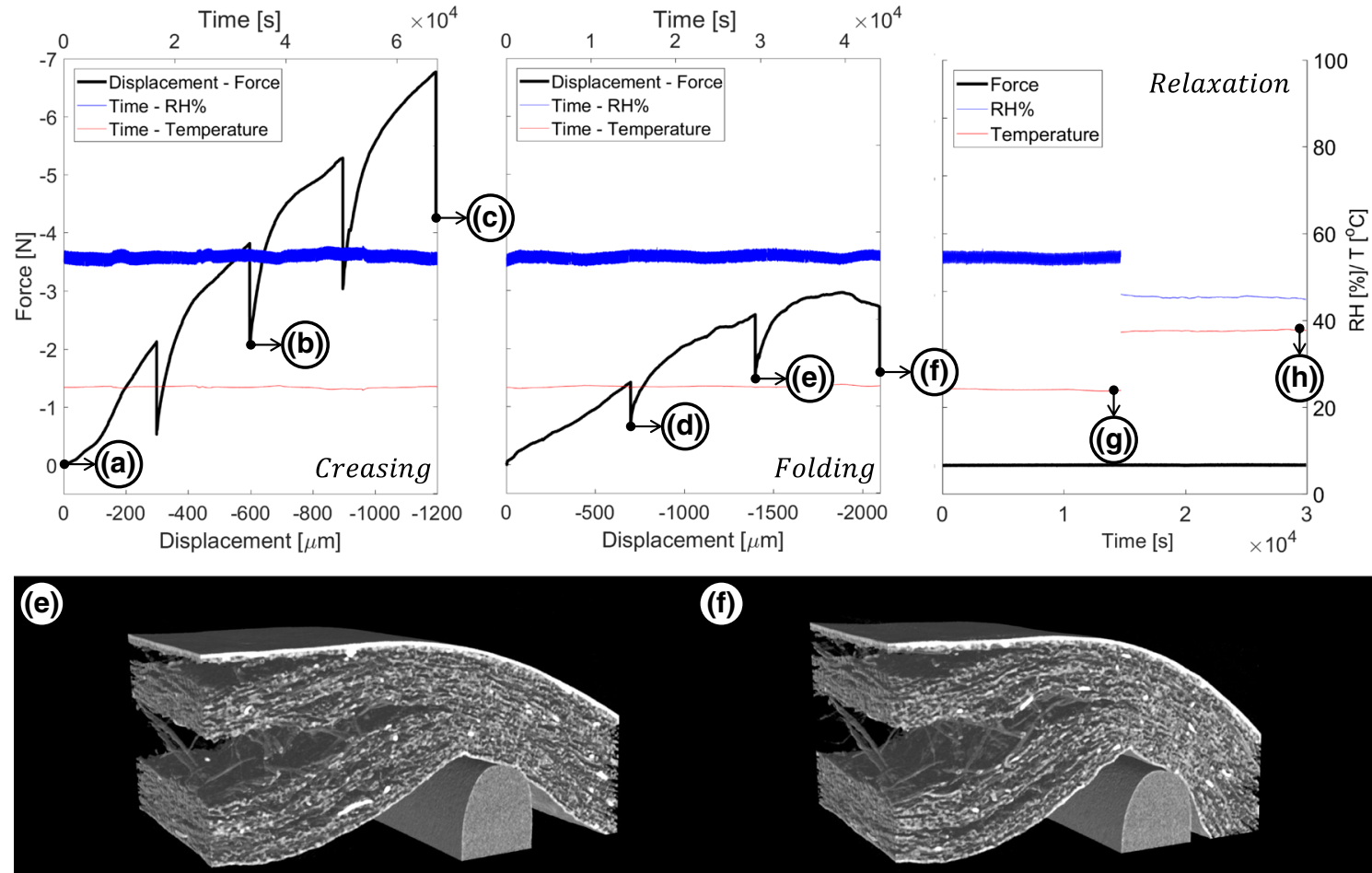

(f)

(g)

(h)
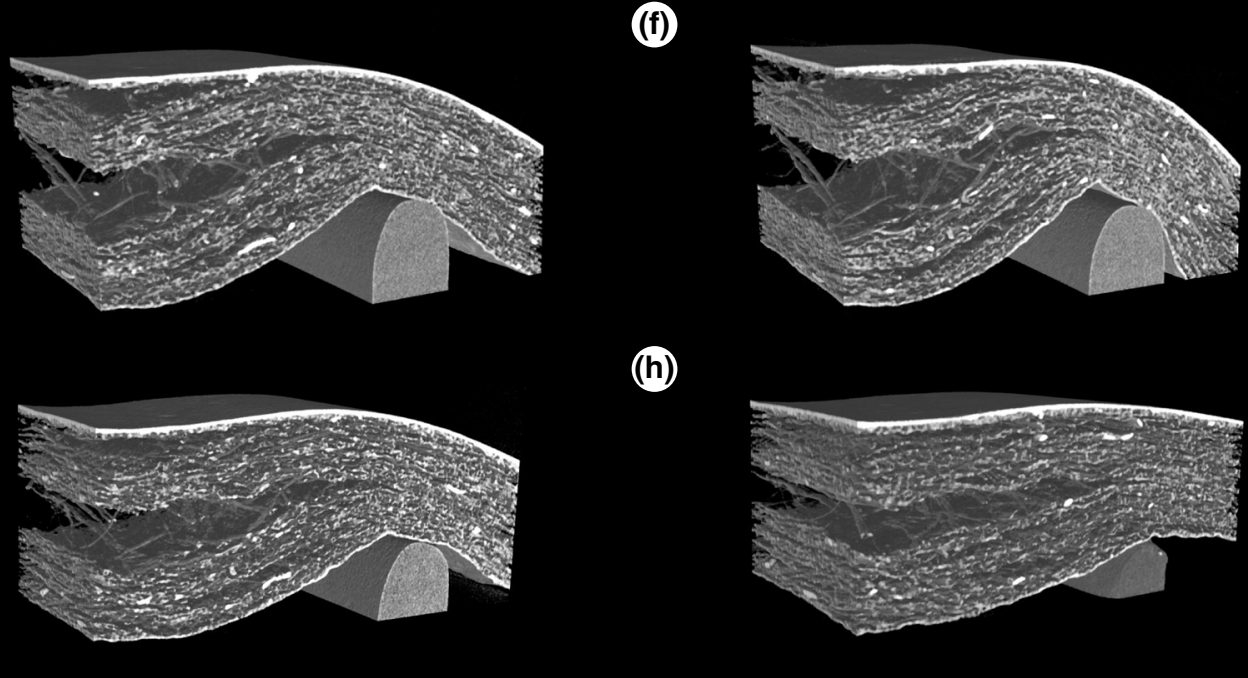

(g)

Fig. 9 Hygro-thermo-mechanical response of the conducted creasing and folding experiments on cardboard with 3D characterizations after: $(a)$ initial configuration, $(b)$ creasing step 2, $(c)$ creasing step 3, $(d)$ creasing step 4, $(e)$ folding step 1, $(f)$ folding step 2, $(g)$ folding step 3, $(g, h)$ relaxation under $(g)$ factory and $(h)$ increased climate conditions. Small-scale delamination of the different layers initiates between the third and fourth creasing step, as shown in $(c)$. After the the first folding step $(d)$, clear separation of the mid-layer from the top layer is visible. After further folding $(f)$, the mid-layer separated into three plies. Individual cross-layer fibres are visible in the folded cardboard 
PMMA to reduce X-ray attenuation. A Nanotom $160 N F$ by General Electric/Phoenix is used to attain full 3D CT characterizations of the intermediate deformations steps during the creasing and folding experiments. The $2 \mathrm{D} X$-ray images were collected with a voltage of $60 \mathrm{kV}$ and a current of $250 \mu \mathrm{A}$. A total of 1400 images were collected with an exposure time of $1250 \mathrm{~ms}$ at an averaging of 6 . An effective voxel size of approximately $3 \mu \mathrm{m}$ was attained to properly capture single paper fibres. Figure $8(c)$ shows 2D X-ray images of the two bending modules with the cardboard sample under load.

The creasing process is done in four increments, $300 \mu \mathrm{m}$ per increment, with a velocity of $2 \mu \mathrm{m} / \mathrm{s}$. Three 3D CT characterizations were conducted during the creasing: before loading and after crease step 2 and 4 . The folding process is done in three increments, $700 \mu \mathrm{m}$ per increment, with a velocity of $2 \mu \mathrm{m} / \mathrm{s}$ and a 3D CT characterization is performed after each bending increment. Both the creasing and folding experiment are conducted in a controlled climate with a temperature and relative humidity of, respectively, $23^{\circ} \mathrm{C}$ and 50\%, which Mayr-Melnhof Eerbeek implied to be the factory processing conditions. Due to this increased relative humidity, continuous relaxation is present in the cardboard sample, which interferes with the long CT scanning time. Hence, after each loading step, the displacement is kept constant for 45 min allowing the sample to relax to a steady state before starting the CT scan. After these two mechanical tests, the force is released for a full day after which another CT scan is performed on the completely relaxed sample. At last, climate accelerated relaxation is conducted at $\mathrm{T}=40{ }^{\circ} \mathrm{C}$ and $\mathrm{RH} \%=45 \%$ for $6 \mathrm{~h}$, and subsequently the final 3D CT characterization is conducted.

Figure 9 shows the hygro-thermo-mechanical response and eight intermittent 3D CT characterizations during the creasing and folding process and after climate conditioning. The images are cropped to a quarter of the full reconstruction to allow better analysis of the intrinsic behaviour and visualization of the failure modes. Based on Fig. 9 two general observations are made that demonstrate the potential of the here-proposed in-situ CT device.

First, the setup allows proper climate-control within acceptable error bounds, while the force, displacement, relative humidity and temperature are accurately monitored. The mechanical response looks similar for each creasing increment, corresponding to plastic deformation of the material. During folding, deviations become visible in the mechanical response, which indicates that failure occurs in the material. This complies with the onset and progression of delamination that is clearly visible in images $(d-h)$. Hence the setup allows direct comparison between the 3D intrinsic behaviour and the mechanical response. The noise level difference on the relative humidity between increments $(a-g)$ and $(h)$ can be explained by the presence of a buffer volume between the climate box and the sample tube, which allows a more constant control of the relative humidity, since the sample tube's volume is rather low.

Second, the 3D CT characterizations allow clear distinction of the multiple layers and full 3D analysis of the occurring failure modes. After creasing increment four (c), the first signs of small scale delamination within the mid-layer becomes visible. During the folding increments, delamination becomes clearly visible, i.e. after increment one $(d)$ the different layers have clearly started to separate from each other. After folding increment three $(f)$, the midlayer is fully separated from the top-layer. Additionally, the mid-layer is completely split up into three separate plies, which was also observed earlier under light microscopy in [12]. The CT characterization of the intermittent increments enables full 3D analysis of the present failure modes. In the current case, the occurring delamination can be analysed up to the fibre level, as cross-layer fibres are clearly visualized between the different plies in images $(d-h)$. Relaxation due to climate change is clearly visible when comparing image $(g)$ with image $(h)$. Moreover, this example nicely demonstrates the CT resolution capabilities for the here-proposed design.

\section{Conclusions and Recommendations}

Being able to perform advanced hygro-thermo-mechanical experiments with intermittent high-quality $\mathrm{CT}$ characterizations opens up a new world for material testing an characterization. A compact, light-weight, climate-controlled, high force and displacement resolution, stationary ROI, multiple loading in-situ CT device has been realised which allows such complex experiments. The device is designed to fit the compact design space of lab-scale CT scanners, particularly the Nanotom $160 \mathrm{NF}$ which puts the highest constraint on the outer geometry and the maximum weight. The conceptual design was based on a comprehensive literature study in which various possible options were discussed. A stationary ROI was realized by using a planetary gear to convert rotational motion into both upand downwards translation. The gears (sun- and ring gear) are connected to a threaded cylinder, which when driven, rotate in opposite direction of each other. Choosing the right pitch for these cylinders allows compensation of the rotational speeds difference of, respectively, sun and ring gear, resulting in equal up- and downwards translation all within the compact design space. Accurate force and displacement measurements are attained by, respectively, allowing exchangeable loadcells and three accurate extensiometers (LVDT's) inside. A maximum stroke of $20 \mathrm{~mm}$ was realized along with a maximum applied load of $2 \mathrm{kN}$, allowing 
testing of a wide variety of materials. A modular clamping method was realized that allows fixation of rectangular and circular samples which require minimal sample geometry adjustments, while additionally, allowing fixation of specific loading modules such as three- and four-point bending clamps. To enable a controlled climate (temperature and relative humidity) around the sample, an external climate box is connected to the sample tube.

Validation experiments were conducted to verify if the device meets the above listed requirements. A limited degree of deterioration of the image quality was observed when scanning the sample inside a tube, however, no additional deterioration was caused by the weight of the setup due to the lightweight rotational symmetric design. Moreover, the CT reconstruction quality was found to be sufficiently high to characterize delamination in cardboard down to the level of individual fibres, which is known to be challenging. Accurate force and displacement measurements are validated by successfully determining the Young's modulus of three brass shafts. Proper climate control inside the sample tube is realized, well within the specified requirements.

The setup's potential is demonstrated by performing the creasing and folding and subsequently relaxation process of cardboard within a climate-controlled environment. Threeand four-point bending clamps are used to, respectively, crease and fold cardboard. Each process is performed in multiple increments and intermittent CT scans on the sample are performed. This allowed full 3D characterization of the material, which can directly be linked to the properly captured mechanical response, allowing identification and analysis of micro-mechanical failure modes, in this case plie delamination during the folding process.

Acknowledgments The authors would like to acknowledge F. Sket for constructive scientific and technological discussions and A. Ruybalid for proofreading.

Open Access This article is distributed under the terms of the Creative Commons Attribution 4.0 International License (http://creativecommons. org/licenses/by/4.0/), which permits unrestricted use, distribution, and reproduction in any medium, provided you give appropriate credit to the original author(s) and the source, provide a link to the Creative Commons license, and indicate if changes were made.

\section{References}

1. Sket F, Enfedaque A, López CD, González C, Molina-Aldareguía J, Llorca J (2016) X-ray computed tomography analysis of damage evolution in open hole carbon fiber-reinforced laminates subjected to in-plane shear. Combust Sci Technol 133:40-50

2. Wismans J, Dommelen JV, Govaert L, Meijer H, Rietberg BV (2009) Computed tomography-based modeling of structured polymers. J Cell Plast 45:157-179
3. Poulet P-A, Hochstetter G, King A, Proudhon H, Joannès S, Laiarinandrasana L (2016) Observations by in-situ X-ray synchrotron computed tomography of the microstructural evolution of semi-crystalline polyamide 11 during deformation. Polym Test. $56: 245-260$

4. Buffiere JY, Maire E, Adrien J, Masse JP, Boller E (2010) In situ experiments with $\mathrm{X}$-ray tomography: an attractive tool for experimental mechanics. Exp Mech 50:289-305

5. Gueninchault N, Proudhon H, Ludwig W (2016) Nanox: a miniature mechanical stress rig designed for near-field X-ray diffraction imaging techniques. J Synchrotron Radiat 23:14741483

6. Bellet D, Gorges B, Dallery J-A, Bernard P, Pereiro E, Baruchel J (2003) A $1300 \mathrm{k}$ furnace for in-situ X-ray microtomography. J Appl Crystallogr 36:366-367

7. Bertrand R, Caty O, Mazars V, Denneulin S, Weisbecker P, Pailhes J, Camus G, Rebillat F (2017) In-situ tensile tests under sem and x-ray computed micro-tomography aimed at studying a self-healing matrix composite submitted to different thermomechanical cycles. J Eur Ceram Soc 37:3471-3474

8. Case R, Schollmeyer H, Kohl P, Sirota EB, Pynn R, Ewert KE, Safinya CR, Li Y (2017) Hydration forces between aligned DNA helices undergoing b to a conformational change: In-situ x-ray fiber diffraction studies in a humidity and temperature controlled environment. J Struct Biol 200:283-292

9. Maksimcuka J, Obata A, Sampson WW, Blanc R, Gao C, Withers P, Tsigkou O, Kasuga T, Lee PD, Poologasundarampillai G (2017) X-ray tomographic imaging of tensile deformation modes of electrospun biodegradable polyester fibers. Front Mater 4:43

10. Chen N, Koker MK, Uzun S, Silberstein MN (2016) Insitu X-ray study of the deformation mechanisms of non-woven polypropylene. Int J Solids Struct 97-98:200-208

11. Bosco E, Bastawrous MV, Peerlings RHJ, Hoefnagels JPM, Geers MGD (2015) Bridging network properties to the effective hygroexpansivity of paper: experiments and modelling. Philos Mag 95:3385-3401

12. Beex LAA, Peerlings RHJ (2009) An experimental and computational study of laminated paperboard creasing and folding. Int $\mathbf{J}$ Solids Struct 46:4192-4207

13. Zhang Y, Lu Z, Yang Z, Zhang D, Shi J, Yuan Z, Liu Q (2017) Compression behaviors of carbon-bonded carbon fiber composites: experimental and numerical investigations. Carbon 116:398-408

14. Arias-Moreno AJ, Ito K, van Rietbergen B (2016) Microfinite element analysis will overestimate the compressive stiffness of fractured cancellous bone. J Biomech 49(13):26132618

15. Sket F, Rodríguez-Hortalá M, Molina-Aldareguía JM, Llorca J, Maire E, Requena G (2015) In situ tomographic investigation of damage development in $\pm 45^{\circ}$ carbon fibre reinforced laminates. Mater Sci Technol 31:587-593

16. General Electronics, phoenix Nanotom m 180 kV/20 W X-ray nanoCT system for high-resolution analysis and 3D metrology, 31344EN (9 2017)

17. General Electronics, phoenix vtomex m Powerful versatile X-ray microfocus CT system for 3D metrology and analysis with up to $300 \mathrm{kV} / 500 \mathrm{~W}, 31319 \mathrm{EN}$ (8 2017)

18. ZEISS, ZEISS Xradia 410 Versa submicron X-ray imaging: bridge the gap in lab-based microscopy, 40011EN (5 2014)

19. Yxlon, YXLON FF35 CT Multi-application, high-resolution computed tomography (CT) inspection system for fine and middle-sized parts, 9499.211.23010.RG13 (5 2014)

20. Deben, In-situ testing stages for XRM/micro-XCT ( 2017) 
21. Shafqat S, Hoefnagels JPM, Savov A, Joshi S, Dekker R, Geers MGD (2017) Ultra-stretchable interconnects for high-density stretchable electronics. Micromachines 8(9):13

22. Wu S, Bie B, Fan D, Sun T, Fezzaa K, Feng Z, Huang J, Luo S (2018) Dynamic shear localization of a titanium alloy under high-rate tension characterized by x-ray digital image correlation. Mater Charact 137:58-66

23. Kumari S, Uphadhyay A, Shukla K (2017) Stress analysis for an infinite plate with circular holes. Mater Today: Proc 4:2323-2332
24. Instron, Small collet grip assembly[online] (2015) [Accessed 29 April. 2018]. URL http://www.instron.us/en-us/products/ testing-accessories/630grips/torsion-axial-torsion-grips/ collet-style/w-mt02

25. Nagasawa S, Fukuzawa Y, Yamaguchi T, Tsukatani S, Katayama I (2003) Effect of crease depth and crease deviation on folding deformation characteristics of coated paperboard. J Mater Process Technol 140(1):157-162 